\title{
Effective Model to Evaluate the Inflow Performance of Highly Deviated Wells in Anisotropic Reservoirs
}

\author{
Xuan Zhao ${ }^{1,2}$, Haijing Wang ${ }^{1, *}$ and Shifeng Xue ${ }^{1}$ \\ ${ }^{1}$ College of Pipeline and Civil Engineering, China University of Petroleum, Qingdao 266580, China \\ ${ }^{2}$ Sinopec International Petroleum Company Colombia Branch, Bogotá, Colombia
}

Received 2 September 2018; Accepted 27 November 2018

\begin{abstract}
Inflow performance evaluation is an important basis for the completion design of highly deviated wells, but existing evaluation models do not consider reservoir anisotropy or well deviation angle with long calculation time and certain limitations. In order to perfect the inflow performance evaluation model of highly deviated wells, a high-efficiency model evaluating their inflow performance in anisotropic reservoirs was proposed in this study. On the basis of a source function method, an oil reservoir-wellbore coupling flow equation was established, and a new model that can evaluate inflow performance of highly deviated wells in anisotropic reservoirs was proposed by introducing the anisotropic quantitative characterization method. On this basis, the influence laws of anisotropy and well deviation angle on inflow morphology, potential section, and productivity of highly deviated wells were discussed. Results indicate that the new model can consider the influences of reservoir anisotropy and well deviation on inflow performance, and the calculation only consumed $1 / 30$ of the traditional model. Reservoir anisotropy has a great influence on the inflow performance of highly deviated wells. The smaller the anisotropy coefficient, the earlier the transient-state flow appears in the transition phase, and the later the steady-state flow appears in the subsequent phases. Anisotropy coefficient has an apparent influence on flow velocity in the wellbore, and flow rates at two ends of the wellbore decrease by $32.3 \%$ when the anisotropy coefficient increases from $1 / 100$ to 100 . The influence of well deviation angle on the highly deviated well production is enhanced as the anisotropy coefficient increases. The study contributes to the highly efficient evaluation of the inflow performance of highly deviated wells and provides a certain reference for the optimization of highly deviated wells and production forecast.
\end{abstract}

Keywords: Highly Deviated, Well Inflow Performance, Reservoir-Wellbore Coupling, Anisotropy

\section{Introduction}

With the development of well drilling and completion technologies, highly deviated wells have been extensively applied to the development of domestic and foreign oil and gas fields [1]. Highly deviated wells can enlarge the contact area between a wellbore and reservoir by a large margin and single-well influence scope, and the mining efficiency is clearly superior to that of a vertical well. Inflow performance evaluation of a highly deviated well is the basis for the optimization design of the well completion scheme and foundation, guaranteeing high recovery ratio. Thus, it has attracted increasing attention from scholars.

However, the wellbore structure of highly deviated wells is totally different from that of vertical wells. A certain angle should be formed between it and the reservoir, so its inflow morphology is sharply different from that of traditional vertical wells [2]. The traditional oil well inflow performance evaluation model is no longer applicable [3]. To establish the inflow performance evaluation method of highly deviated wells, a new oil reservoir-wellbore flow model must be established. Moreover, given the influence laws of factors, such as reservoir anisotropy, well deviation

*E-mail address: zhuxx99@126.com

ISSN: $1791-2377$ @ 2018 Eastern Macedonia and Thrace Institute of Technology. All rights reserved. doi:10.25103/iestr.115.14 angle, and wellbore nearside manifold, many involved works encounter great technological difficulties.

Thus, scholars have carried out many studies on the inflow performance evaluation method of highly deviated wells [4-8]. However, main works concentrate on using source function method to analyze flow dynamics and potential energy distribution of highly deviated wells under non-steady flow, but no in-depth study has been implemented on problems, such as how reservoir anisotropy coefficient and well deviation angle influence the flow pattern of highly deviated wells and how to process the anisotropy coefficient in the evaluation model. In addition, the calculation process of flow dynamics evaluation of the highly deviated well consumes too much time, which restricts popularization and application of highly deviated wells. Therefore, how to establish an oil reservoir-wellbore flow pattern that is applicable to highly deviated wells, determine the influence laws of reservoir anisotropy and well deviation angle, and propose a high-efficiency model evaluating the inflow performance of highly deviated wells constitute a problem that needs urgent solutions.

Therefore, a new-type oil reservoir-wellbore coupling flow model was established by improving the source function method in this study. In accordance with the accuracy and efficiency verification of the model, the influence laws of reservoir anisotropy coefficient and well deviation angle on the inflow performance of highly 
deviated wells and their features in terms of inflow morphology, inflow section, potential section, and productivity evaluation were studied. This study aims to propose a set of high-efficiency evaluation model, which considers the influences of reservoir anisotropy and well deviation angle, and to provide a reference for the optimization design of the well completion scheme of highly deviated wells.

\section{State of the art}

Existing inflow performance evaluation methods on isotropic horizontal or vertical wells are quite mature. However, with the extensive application of highly deviated wells, the traditional evaluation methods are no longer applicable because they do not consider the influences of reservoir anisotropy and well deviation. Thus, scholars have carried out many works on evaluation model and algorithm of inflow performance of highly deviated wells. Vaferia [9] and Biryukov [10] studied the inflow performance of vertical and horizontal wells with simple features of reservoir model and linear planning technology and mentioned study difficulties of the inflow performance of highly deviated wells. However, no in-depth study was implemented. Bahadori [11] developed a simple oil flow prediction equation as the dimensionless length of the leakage flow area to simulate deviated wells, but this equation did not provide the solution of transient-state pressure. Ahmadi [12] used artificial neural network method to develop a vector model to predict pseudo-skin factor of wells in a rectangular leakage flow area and evaluated oil well productivity. However, this method had a tedious process and did not consider reservoir anisotropy. Tabatabaei [13] first considered the three different boundary conditions of dynamic inflow analysis and prediction. He studied the internal qualitative relationship between transient-state pressure behaviors and inflow performance of the wells, but he did not establish any inflow performance evaluation model. To consider the influences of anisotropy and bottom water reservoir features on oil well productivity, Shi T [14] and Wang Y N [15] proposed a new inflow performance model and productivity prediction method, but this method was more suitable for horizontal well and did not consider well deviation. Therefore, Chen Y Q [16] and Lei G [17] further studied and analyzed pressure features of the anisotropic oil well and gave the inflow performance models of horizontal and highly deviated wells. However, the model only considered non-steady flow of the oil well, and model boundary was set as a non-flow boundary, which cannot reflect the real features of non-transient flow of the oil well. Soleimani [18] deemed that well deviation has a minor influence on the productivity of the homogeneous oil reservoir but has significant influence on the productivity of heterogenous and anisotropic oil reservoirs. He corrected non-steady flow transient pressure to solve the model of the highly deviated well, but the model did not analyze infiniteconductivity reservoirs. Delalat [19] studied the influences of reservoir homogeneity on inflow performance and deemed that homogeneity characteristic can significantly reduce the productivity of highly deviated wells. Therefore, considering reservoir anisotropy, Williams [20] proposed a wellbore-oil reservoir coupling model to study the inflow performance of an unconventional well within a well deviation angle range of $0^{\circ}-90^{\circ}$, but this method still did not consider the status of infinite-conductivity reservoirs. Wang
H J [21] used source function and coordinate transformation methods to establish an inflow model of a highly deviated well at anisotropic oil reservoirs and analyzed the influence laws of reservoir anisotropy and well deviation angle. However, the calculation process of this model was timeconsuming and has low calculation efficiency. Therefore, Fang S D [22] and Ren Z X [23] studied inflow performance algorithm and tried to seek a highly efficient inflow performance evaluation method, but its effect was not ideal.

The studies were mainly directed at inflow performance of non-steady flow highly deviated wells, but few studies were applicable to the inflow performance of extensively used finite-conductivity highly deviated wells, as well as high-efficiency evaluation models of inflow performance considering anisotropy and well deviation. The improved transient source function method was used to establish an oil reservoir-wellbore coupling flow model suitable for the finite-conductivity highly deviated well, which can effectively shorten calculation time. On this basis, the transient pressure distribution of finite-conductivity highly deviated wells in anisotropic reservoirs was analyzed along with the influence laws of two key parameters - anisotropy and well deviation angle-on inflow morphology, inflow section, potential section, and productivity, laying a foundation for well completion optimization design of highly deviated wells.

The remainder of this study is organized as follows. The new oil reservoir-wellbore coupling flow model suitable for a finite-conductivity highly deviated well is described and the model effectiveness is verified in Section 3. Inflow performance analysis of an incomplete highly deviated well is taken as an example and influence laws of anisotropy and well deviation on flow morphology, potential section, and productivity are studied in Section 4. The present study is summarized, and relevant conclusions are drawn in the final section.

\section{Methodology}

\subsection{New-type inflow performance evaluation model}

Figure 1 shows a schematic diagram of an oil reservoirwellbore system structure. The highly deviated well is located in the parallel hexahedron reservoir, and the external boundary of the oil reservoir is of constant pressure or impermeable. Reservoir thickness is $h$, porosity is $\phi$, and permeability coefficients in three directions are $K_{x}, K_{y}$ and $K_{z}$. The radius, length, and deviation angle of the highly deviated well are $r_{w}, l$ and $\theta_{w}$, respectively. The center of the well is located at $z_{w}$ above the lower boundary. Singlephase and micro compressible fluid in the oil reservoir flows at an initial uniform potential.

\subsubsection{Traditional inflow performance evaluation model}

As a traditional method studying transient-state behaviors of well pressure, source function method regards the wellbore as a continuous line source/sink on the borehole axis, and pressure response is obtained through the integrals of transient point source on well trajectory and time. For any point $M(x, y, z)$ and time $t$, the fluid potential change caused by slope line sink $\mathrm{S}$ can be expressed as follows: 
$\Delta \phi(M, S, t)=\int_{0}^{t} \int_{S} \frac{q}{\phi c} G_{P}\left(M, M_{w}, t-\tau\right) d l d \tau$

where $\Delta \phi$ is the potential drawdown, which is defined ${ }^{\text {as }}$ Error! Objects cannot be created from editing field codes.; $p$ is the pressure; $\rho$ is the fluid density; $g$ is the acceleration of gravity; $q$ is the inflow rate per unit wellbore length; $\phi$ is the reservoir porosity; $c$ is the comprehensive compression factor; and $G_{p}\left(M, M_{w}, t\right)$ describes the transient source function of point source $M_{w}$ along line segment $S$ and can be acquired through the product of corresponding one-dimensional source function. For example, thickness is $z_{e}$, the upper and lower boundaries are located in the infinite-plate oil reservoir with impermeable boundary, and point source transient source function at $M_{w}\left(x_{w}, y_{w}, z_{w}\right)$ can be expressed as:

$$
\begin{aligned}
G_{\mathrm{p}}\left(M, M_{\mathrm{w}}, t\right)= & \exp \left[\frac{\left(x-x_{\mathrm{w}}\right)}{4 \chi t}\right] / \sqrt{4 \pi \chi t} \cdot \exp \left[\frac{\left(y-y_{\mathrm{w}}\right)}{4 \chi t}\right] / \sqrt{4 \pi \chi t} \\
& \cdot \frac{1}{z_{\mathrm{e}}}\left[1+2 \sum_{n=1}^{\infty} \exp \left(-\frac{n^{2} \pi^{2} \chi t}{z_{\mathrm{e}}{ }^{2}}\right) \cos \frac{n \pi z_{\mathrm{w}}}{z_{\mathrm{e}}} \cos \frac{n \pi z}{z_{\mathrm{e}}}\right]
\end{aligned}
$$

where $\chi$ is the hydraulic diffusivity constant.

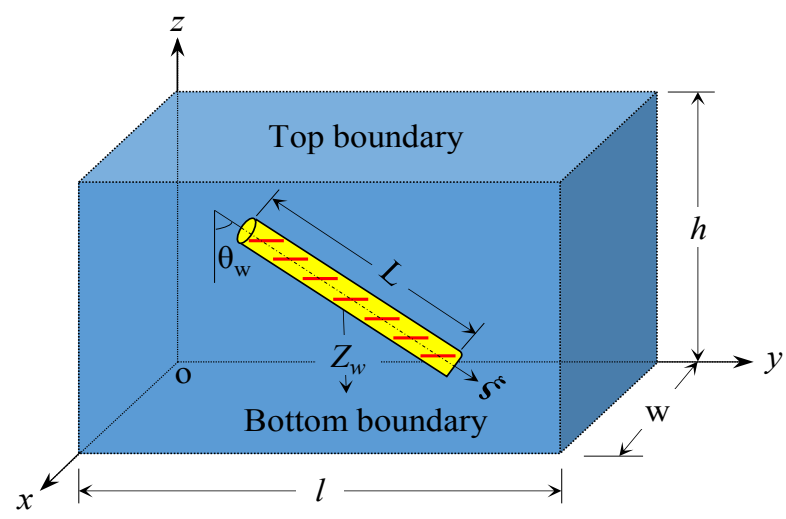

Fig. 1. Discrete model of a slanted well in a parallelepiped reservoir

Actual inflow rate $q$ changes with the well wellbore, which is a function of time. Therefore, obtaining the analytical expression of inflow rate is very difficult. For approximate calculation, space and time are usually dispersed, time interval $0: t$ is divided into $m$ subintervals $(j=1,2, \ldots \ldots . m)$, and borehole axis is divided into $n$ segments $s_{i}(i=1,2, \ldots, n)$. At time $j$, the inflow rate of unit length of the $i(t h)$ well segment is a constant, so Equation (1) is converted into:

$\Delta \Phi(M, S, t)=\sum_{j=1}^{\mathrm{m}} \sum_{i=1}^{\mathrm{n}} \frac{q_{i}^{j}}{\phi c} \int_{t_{j-1}}^{t_{j}} \int_{\mathrm{S}_{i}} G_{\mathrm{P}}\left(M, M_{\mathrm{w}}, t-\tau\right) \mathrm{d} l \mathrm{~d} \tau$

As transient point source function $G_{p}\left(M, M_{w}, t\right)$ in the bounded space involves infinite series, the calculation of Equation (3) becomes time-consuming, which hinders the actual application of this method.

\subsubsection{New-type inflow performance evaluation model}

The traditional inflow evaluation model was perfected in this study through the improved source function method. Combined with coordination transformation method, a newtype inflow performance evaluation model was proposed. This model can consider reservoir anisotropy with clearly elevated calculation speed. The perfection method of the inflow performance evaluation model is as follows:

First, each slope line sink $S_{i}$ is replaced by a horizontal $\left(\theta_{w} \geq 45^{\circ}\right)$ or vertical $L_{i}\left(\theta_{w}<45^{\circ}\right)$. New and original line sinks have the same midpoint and length. Thus, Equation (3) can be converted into:

$$
\Delta \Phi(M, S, t)=\sum_{j=1}^{m} \sum_{i=1}^{n} \frac{q_{i}^{j}}{\phi c} \int_{t_{j-1}}^{t_{j}} \int_{\mathrm{L}_{i}} G_{\mathrm{P}}\left(M, M_{\mathrm{w}}, t-\tau\right) \mathrm{d} l \mathrm{~d} \tau
$$

Second, fluid potential at midpoint of each segment will nearly remain unchanged only if micro-segment length $\Delta L_{i}(i=1,2 \ldots ., n)$ is sufficiently small compared with reservoir dimensions. Saint-Venant principle indicates that this approximation only influences the area near the microsegment. Micro-segment $L_{i}$ is parallel to the boundary. Thus, transient point source function $G_{p}\left(M, M_{w}, t\right)$ is the integral of transient linear source function $G_{L}(M, L i, t)$ on microsegment $L_{i}$, and it can be easily obtained using the existing one-dimensional transient source function and Newman multiplication method. The transient source function for a horizontal line source $L$ with length is as follows:

$$
\begin{aligned}
G_{L}(M, L, t)= & \frac{1}{2}\left\{\operatorname{erf}\left[\frac{x_{\mathrm{f}} / 2+\left(x-x_{\mathrm{w}}\right)}{\sqrt{4 \chi t}}\right]+\operatorname{erf}\left[\frac{x_{\mathrm{f}} / 2-\left(x-x_{\mathrm{w}}\right)}{\sqrt{4 \chi t}}\right]\right\} \\
& \cdot \exp \left[\frac{\left(y-y_{\mathrm{w}}\right)}{4 \chi t}\right] / \sqrt{4 \pi \chi t} \\
& \cdot \frac{1}{z_{\mathrm{e}}}\left[1+2 \sum_{n=1}^{\infty} \exp \left(-\frac{n^{2} \pi^{2} \chi t}{z_{\mathrm{e}}^{2}}\right) \cos \frac{n \pi z_{\mathrm{w}}}{z_{\mathrm{e}}} \cos \frac{n \pi z}{z_{\mathrm{e}}}\right]
\end{aligned}
$$

Therefore, Equation (4) can be simplified as follows:

$$
\Delta \Phi(M, S, t)=\sum_{j=1}^{m} \sum_{i=1}^{n} \frac{q_{i}^{j}}{\phi c} \int_{t_{j-1}}^{t_{j}} G_{L}\left(M, L_{i}, t-\tau\right) \mathrm{d} \tau
$$

Through a comparison of Equations (6) and (4), the double integral in Equation (4) was converted into unilabiate functional integral in Equation (6). Thus, the simplified model can significantly improve calculation efficiency.

Actual reservoir is anisotropic, that is, permeability anisotropy has a great influence on the inflow performance of a highly deviated well. To solve reservoir anisotropy problem, the following coordinate transformation is defined:

$$
x^{\prime}=\frac{\sqrt{K_{y} K_{z}}}{\sqrt[3]{K_{x} K_{y} K_{z}}} x, \quad y^{\prime}=\frac{\sqrt{K_{x} K_{z}}}{\sqrt[3]{K_{x} K_{y} K_{z}}} y, \quad z^{\prime}=\frac{\sqrt{K_{x} K_{y}}}{\sqrt[3]{K_{x} K_{y} K_{z}}} z
$$

Actual anisotropic reservoir can be transformed into an equivalent isotropic system with effective permeability $K^{\prime}=\sqrt[3]{K_{x} K_{y} K_{z}}$. After the coordinate transformation, the relative position of the wellbore in the reservoir remains unchanged. In addition, flow history and 
pressure response of point $M(x, y, z)$ in the original system are the same as those of point $M^{\prime}\left(x^{\prime}, y^{\prime}, z^{\prime}\right)$ in the equivalent isotropic system. Fluid potential change in the anisotropic oil reservoir can be obtained as:

$$
\Delta \Phi(M, S, t)=\Delta \Phi\left(M^{\prime}, S^{\prime}, t\right)=\sum_{j=1}^{\mathrm{m}} \sum_{i=1}^{\mathrm{n}} \frac{q_{i}^{j} \Delta L_{i}}{\phi c \Delta L_{i}^{\prime}} \int_{t_{j-1}}^{t_{j}} G_{L}\left(M^{\prime}, L_{i}^{\prime}, t-\tau\right) \mathrm{d} \tau
$$

Where $\Delta L_{i}^{\prime}$ and $\Delta L_{i}$ are the lengths of micro-segment $i$ in the equivalent anisotropic system and original system, respectively.

$M_{w f, I}$ is the micro-segment midpoint, and its distance from the line sink is $r_{w}$. Borehold fluid potential change of each micro-segment can be obtained as follows:

$$
\Delta \Phi_{\mathrm{w}, i}=\sum_{k=1}^{\mathrm{m}} \sum_{j=1}^{\mathrm{n}} \frac{q_{j}^{k} \Delta L_{j}}{\phi c \Delta L_{j}^{\prime}} \int_{t_{k-1}}^{t_{k}} G_{\mathrm{L}}\left(M_{\mathrm{wf}, i}{ }^{\prime}, L_{j}^{\prime}, t-\tau\right) \mathrm{d} \tau(i=1,2, \ldots . n)
$$

Therefore, borehole fluid potential of each microsegment can be obtained as:

$$
\begin{aligned}
& \Phi_{\mathrm{w}, i}=\Phi_{0}-\sum_{k=1}^{m-1} \sum_{j=1}^{n} \frac{q_{j}^{k} \Delta L_{j}}{\phi c \Delta L_{j}^{\prime}} \int_{t_{k-1}}^{t_{k}} G_{\mathrm{L}}\left(M_{\mathrm{wf}, i}{ }^{\prime}, L_{j}^{\prime}, t-\tau\right) \mathrm{d} \tau \\
& -\sum_{j=1}^{\mathrm{n}} \frac{q_{j} \Delta L_{j}}{\phi c \Delta L_{j}^{\prime}} \int_{t_{\mathrm{m}-1}}^{t} G_{\mathrm{L}}\left(M_{\mathrm{wf}, i}{ }^{\prime}, L_{j}^{\prime}, t-\tau\right) \mathrm{d} \tau(i=1,2, \ldots . . n)
\end{aligned}
$$

When the time is sufficient and inflow performance is stable, the borehole fluid potential is

$$
\Phi_{\mathrm{w}, i}=\Phi_{0}-\sum_{j=1}^{n} \frac{q_{i} \Delta L_{i}}{\phi c \Delta L_{i}^{\prime}} \int_{0}^{t} G_{\mathrm{L}}\left(M_{\mathrm{wf}, i}^{\prime}, L_{j}^{\prime}, t-\tau\right) \mathrm{d} \tau(i=1,2, \ldots, n)
$$

\subsubsection{Verification of the new-type inflow performance evaluation model}

In this study, the highly deviated well in the impermeableboundary isotropic infinite-plate reservoir was taken as an example, and traditional and new-type inflow performance evaluation models were used to calculate transient pressure response of equivalent nodes in the wellbore. Accuracy and high efficiency of the new-type inflow performance evaluation model were verified. In the calculated example, the highly deviated well was divided into two working conditions: incomplete well and complete well.

To ensure that the equivalent pressure point is located at the midpoint of the micro-segment, the wellbore was divided into 21 micro-segments with length $L / 20$ except for two micro-segments at the well end.

The dimensionless variables are defined as follows:

$$
\begin{aligned}
& \Phi_{\mathrm{D}}=\frac{2 \pi K h\left(\Phi_{0}-\Phi\right)}{\mu Q}, t_{\mathrm{D}}=\frac{K t}{\phi \mu c r_{\mathrm{w}}^{2}}, h_{\mathrm{D}}=\frac{h}{r_{\mathrm{w}}}, \\
& z_{\mathrm{wD}}=\frac{z_{\mathrm{w}}}{r_{\mathrm{w}}}, L_{\mathrm{D}}=\frac{L}{r_{\mathrm{w}}},
\end{aligned}
$$

where $K$ is the permeability of the isotropic oil reservoir, $\Phi_{0}$ is the initial oil reservoir potential, $\mu$ is the fluid viscosity, and $Q$ is the yield.
The calculation results of the new-type inflow performance and traditional models are presented in Tables 1 and 2. Table 1 lists wellbore pressure responses at different times under incomplete well condition, where dimensionless thickness $h_{D}$ and wellbore length $L_{D}$ are both 1,000, and five well deviation angles $\theta_{w}=0^{\circ}, 30^{\circ}, 60^{\circ}, 75^{\circ}$, and $90^{\circ}$ are included.

Table 2 lists the internal pressure responses of the wellbore at different times under complete well condition when $h_{D}=1,000$. Table 2 shows that the results of the newtype inflow performance evaluation are identical with those of the traditional model. In terms of calculation time, the new-type model is only $1 / 30$ of the traditional model. Therefore, the new-type model significantly improves calculation efficiency on the precondition that the calculation accuracy is guaranteed.

\subsection{Oil reservoir-wellbore coupling flow model}

To perfect the inflow performance model of the finiteconductivity highly deviated well, the oil reservoir-wellbore coupling flow model, which can coordinately consider the interaction between reservoir inflow and wellbore flow, is established in this section.

Equation (10) describes the influence of intra-reservoir flow on the fluid potential in the wellbore. Wellbore potential distribution is also influenced by fluid flow in the wellbore. The interaction between reservoir inflow and wellbore flow is quite complicated. Fluid flow in the wellbore is different from fluid flow inside a conventional pipeline because radial inflow quantity is controlled by wall friction and change of kinetic energy. The potential gradient of single-phase flow is

$$
\frac{\mathrm{d} \Phi}{\mathrm{d} x}=-\tau_{\mathrm{w}} \frac{S}{A}-\rho \frac{\mathrm{d}}{\mathrm{dx}}\left(\frac{v}{\lambda}\right)^{2}
$$

where $\tau_{w}$ is the frictional shear stress, $S$ is the wetted perimeter of the wellbore, $A$ is the through-flow area of the wellbore, and $\lambda$ is the momentum correction coefficient.

According to the consistency between well mouth potential and flow velocity, the discrete form of Equation (13) is

$$
\Phi_{\mathrm{w}, i}-\Phi_{\mathrm{w}, i+1}=-\frac{\rho f}{2 d}\left(\frac{v_{i}+v_{i+1}}{2}\right)_{(i=1,2, \ldots \ldots, n-1)}^{2} \Delta L_{i}-\frac{\rho}{\lambda}\left(v_{i}^{2}-v_{i+1}^{2}\right)
$$

where $f$ is the Fanning frictional coefficient, $d$ is the inner diameter of casing pipe, and $v_{i}$ is the average velocity of midpoint of the $i(t h)$ segment on the cross section, which is determined according to Equation (15).

$$
v_{i}=\left(\sum_{j=i}^{n} q_{j}-0.5 q_{i}\right) / A(i=1,2, \ldots \ldots ., n-1)
$$

Therefore, Equations (10) and (14) couple wellbore potential models of reservoir and highly deviated well. Determining the flux and potential distribution along the wellbore requires solving the two equations simultaneously. 
Table 1. Solution comparison of simplified and conventional models for highly deviated, partially penetrating wells $\left(h_{D}=1000, L_{D}=1000\right.$, and $\left.Z_{W D}=500\right)$

\begin{tabular}{|c|c|c|c|c|c|c|c|c|c|c|c|}
\hline \multirow{2}{*}{\multicolumn{2}{|c|}{$\theta_{w}\left({ }^{0}\right)$}} & \multicolumn{10}{|c|}{$t_{D}$} \\
\hline & & $10^{-1}$ & $10^{\circ}$ & $10^{1}$ & $10^{2}$ & $10^{3}$ & $10^{4}$ & $10^{5}$ & $10^{6}$ & $10^{7}$ & $10^{8}$ \\
\hline \multirow{2}{*}{0} & Conventional & 0.012 & 0.522 & 1.568 & 2.708 & 3.858 & 5.009 & 6.160 & 7.312 & 8.463 & 9.614 \\
\hline & Simplified & 0.012 & 0.522 & 1.56 & 2.709 & 3.860 & 5.012 & 6.165 & 7.318 & 8.470 & 9.623 \\
\hline \multirow{2}{*}{30} & Conventional & 0.012 & 0.522 & 1.568 & 2.708 & 3.858 & 4.966 & 6.048 & 7.160 & 8.307 & 9.458 \\
\hline & Simplified & 0.012 & 0.522 & 1.568 & 2.700 & 3.813 & 4.941 & 6.000 & 7.114 & 8.261 & 9.413 \\
\hline \multirow{2}{*}{60} & Conventional & 0.012 & 0.522 & 1.568 & 2.708 & 3.858 & 4.989 & 5.938 & 6.975 & 8.112 & 9.262 \\
\hline & Simplified & 0.011 & 0.522 & 1.568 & 2.700 & 3.813 & 4.939 & 5.888 & 6.927 & 8.065 & 9.216 \\
\hline \multirow{2}{*}{75} & Conventional & 0.012 & 0.522 & 1.568 & 2.708 & 3.858 & 4.989 & 5.916 & 6.925 & 8.059 & 9.209 \\
\hline & Simplified & 0.015 & 0.522 & 1.568 & 2.705 & 3.846 & 4.976 & 5.904 & 6.915 & 8.049 & 9.200 \\
\hline \multirow{2}{*}{90} & Conventional & 0.011 & 0.522 & 1.568 & 2.708 & 3.858 & 4.989 & 5.910 & 6.909 & 8.041 & 9.191 \\
\hline & Simplified & 0.012 & 0.522 & 1.568 & 2.709 & 3.860 & 4.992 & 5.914 & 6.914 & 8.047 & 9.198 \\
\hline
\end{tabular}

Table 2. Solution comparison of simplified and conventional models for highly deviated, fully penetrating wells $\left(h_{D}=1000\right)$

\begin{tabular}{|c|c|c|c|c|c|c|c|c|c|c|c|}
\hline \multirow{2}{*}{\multicolumn{2}{|c|}{$\theta_{w}\left({ }^{0}\right)$}} & \multicolumn{10}{|c|}{$t_{D}$} \\
\hline & & $10^{-1}$ & $10^{\circ}$ & $10^{1}$ & $10^{2}$ & $10^{3}$ & $10^{4}$ & $10^{5}$ & $10^{6}$ & $10^{7}$ & $10^{8}$ \\
\hline \multirow{2}{*}{45} & Conventional & 0.008 & 0.369 & 1.108 & 1.915 & 2.728 & 3.548 & 4.462 & 5.483 & & \\
\hline & Simplified & 0.008 & 0.369 & 1.108 & 1.914 & 2.679 & 3.4848 & 4.397 & 5.420 & & \\
\hline \multirow{2}{*}{60} & Conventional & 0.006 & 0.261 & 0.784 & 1.3542 & 1.9293 & 2.508 & 3.211 & 4.073 & 5.170 & \\
\hline & Simplified & 0.006 & 0.261 & 0.784 & 1.354 & 1.915 & 2.483 & 3.186 & 4.049 & 5.147 & \\
\hline \multirow{2}{*}{75} & Conventional & 0.003 & 0.135 & 0.405 & 0.701 & 0.998 & 1.297 & 1.687 & 2.248 & 3.200 & \\
\hline & Simplified & 0.003 & 0.1352 & 0.405 & 0.701 & 0.998 & 1.294 & 1.684 & 2.246 & 3.198 & \\
\hline \multirow{2}{*}{85} & Conventional & 0.001 & 0.045 & 0.136 & 0.236 & 0.336 & 0.437 & 0.568 & 0.795 & 1.325 & 2.286 \\
\hline & Simplified & 0.001 & 0.045 & 0.136 & 0.236 & 0.336 & 0.437 & 0.568 & 0.795 & 1.326 & 2.287 \\
\hline \multirow{2}{*}{90} & Conventional & 0.000 & 0.009 & 0.027 & 0.047 & 0.067 & 0.087 & 0.113 & 0.159 & 0.293 & 0.671 \\
\hline & Simplified & 0.000 & 0.009 & 0.027 & 0.047 & 0.067 & 0.087 & 0.113 & 0.159 & 0.293 & 0.671 \\
\hline
\end{tabular}

\section{Result analysis and discussion}

On the basis of the new-type oil reservoir inflow performance evaluation model established in Section 3.1, the inflow performance of finite-conductivity highly deviated well in the anisotropic reservoir was analyzed in this study. Influence laws of two major factors-anisotropy and well deviation angle - on inflow morphology, inflow section, and wellbore potential section and oil well productivity of highly deviated wells were studied.

Table 3. Basic well and reservoir characteristics

\begin{tabular}{l|l}
\hline Parameters & Value \\
\hline Reservoir length, $m$ & 300 \\
Reservoir width, $m$ & 300 \\
Reservoir thickness, $m$ & 50 \\
Reservoir equivalent permeability, $\mathrm{\mu m}^{2}$ & 1 \\
Reservoir porosity & 0.3 \\
Initial reservoir potential, $\mathrm{MPa}$ & 10 \\
Fluid density, $\mathrm{kg} / \mathrm{m}^{3}$ & 850 \\
Fluid viscosity, $\mathrm{mPa} \cdot \mathrm{s}$ & 10 \\
Fluid volume factor & 1.25 \\
Comprehensive compression factor, $\mathrm{MPa}^{-1}$ & $1.0 \times 10^{-3}$ \\
Wellbore inclination angle, ${ }^{-3}$ & 45 \\
Wellbore radius, $m$ & 0.1 \\
Wellbore length, $m$ & 50 \\
Relative roughness of casing & 0.001 \\
\hline
\end{tabular}

The edge water-driven parallel hexahedron reservoir shown in Fig. 1 was taken as an example. The upper and lower and left and right boundaries were all set as impermeable boundaries, pressures of front and back boundaries were set as the initial reservoir pressure, and the reservoir presents transverse isotropy. The ideal incomplete highly deviated barefoot well is located in the center of the oil reservoir, which carries out production at a constant rate of $100 \mathrm{~m}^{3} / \mathrm{d}$.

Table 3 lists the characteristic parameters of reservoir, fluid, and wellbore. To maintain a constant equivalent permeability, five different anisotropic coefficients were introduced, namely, 1/100, 1/10, 1, 10, and 100 .

The equivalent isotropic system of the initial anisotropic physical model can be obtained through the coordinate transformation. Table 4 lists the dimensions of oil reservoir and wellbore. Table 4 shows that strata thickness increases after the transformation as the anisotropy coefficient increases, whereas ratios of well deviation angle and wellbore length after the transformation to oil reservoir thickness decrease.

\subsection{Flow regime}

Figure 2 shows the change curves of wellbore heel end potential with time. The result indicates three flow regimes in the wellbore: early-stage radial flow, transition-stage transient flow, and later-stage steady flow. Before the boundary effect appears at the early stage, radial flow occurs in the plane vertical to well axis. During the period of radial flow, the potential logarithmic time curve is a straight line with the slope of $1.15 h^{\prime} / h_{w}^{\prime}$. As the anisotropy coefficient decreases, the duration of radial flow is shortened. With the appearance of boundary effect, transition flow appears earlier and duration is longer. At the later stage, the influences of top and bottom boundaries tend to be stable, flow in the reservoir is mainly steady horizontal flow, and potential logarithmic time curve changes into a horizontal line. Pseudo-radial flow starts as the anisotropy coefficient reduces. 
Table 4. Transformed dimensions of reservoir and wellbore

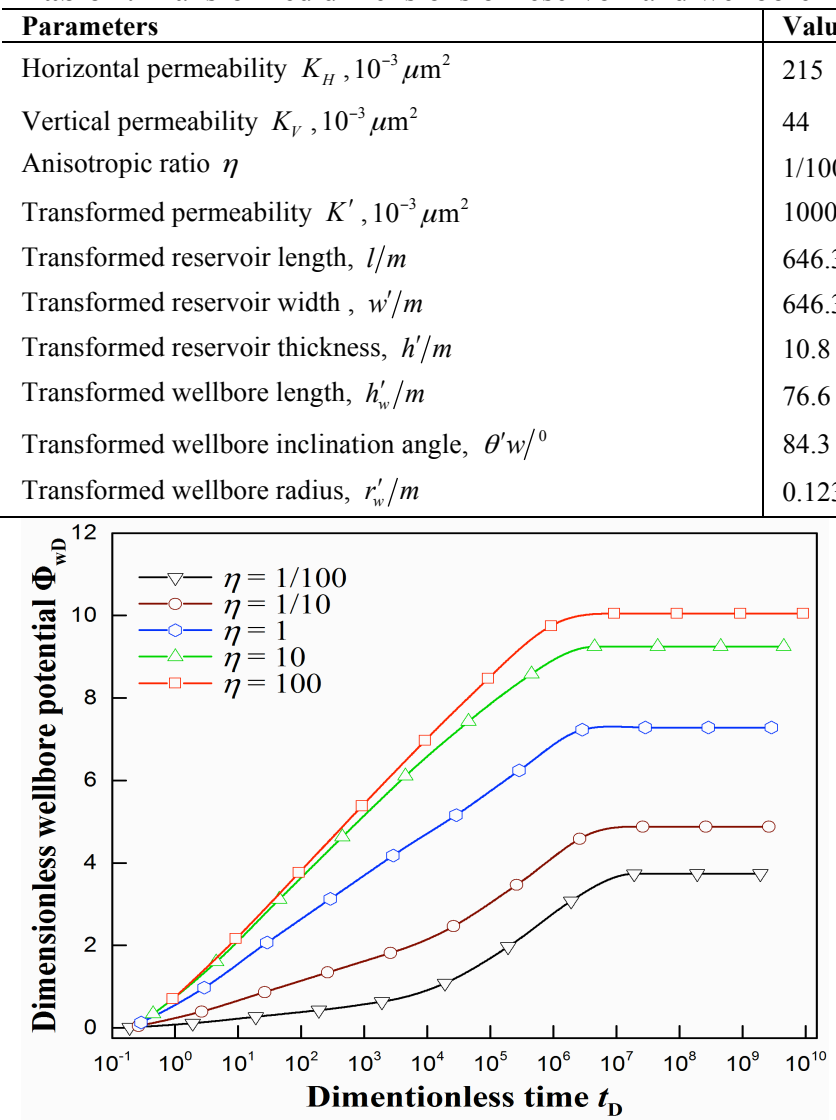

Fig. 2. Changes in bottom-hole potential drawdown with time

\subsection{Inflow-potential distribution}

$10^{-6} d$ potential section and inflow distribution under radial flow conditions are shown in Figs. 3 and 4. The permeability in the direction vertical to borehole axis has a significant influence on wellbore bottom potential. A comparison between potential in Fig. 3 and transformation scale in Table 4 shows that in the equivalent isotropic system, wellbore bottom potential increases as wellbore length and borehole radius increase after the transformation. Influenced by wall frictional pressure drop and accelerated pressure drop in the wellbore, the heel-end potential is lower than the toe-end potential. In addition, pressure drop in the reservoir is more significant than the potential drop, so its influence on inflow distribution is more significant. Figure 4 shows that inflow rate gradually increases from toe end to heel end. The inflow difference between toe end and heel end increases as the potential decreases.

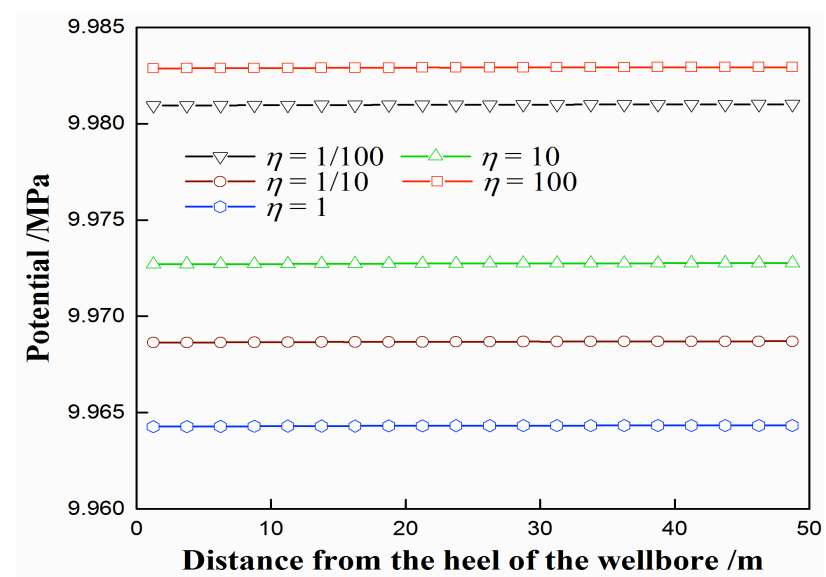

Fig. 3. Wellbore potential profiles at $10^{-6} d$

\begin{tabular}{l|l|l|l|l} 
Value & 464 & 1000 & 2154 & 4641 \\
44 & 4641 & 1000 & 215 & 46 \\
100 & $1 / 10$ & 1 & 10 & 100 \\
000 & 1000 & 1000 & 1000 & 1000 \\
46.3 & 440.3 & 300.0 & 204.4 & 139.2 \\
46.3 & 440.3 & 300.0 & 204.4 & 139.2 \\
0.8 & 23.2 & 50.0 & 107.7 & 232.1 \\
6.6 & 54.4 & 50.0 & 79.9 & 164.9 \\
4.3 & 72.5 & 45.0 & 17.5 & 5.7 \\
.123 & 0.105 & 0.100 & 0.080 & 0.056 \\
\hline
\end{tabular}

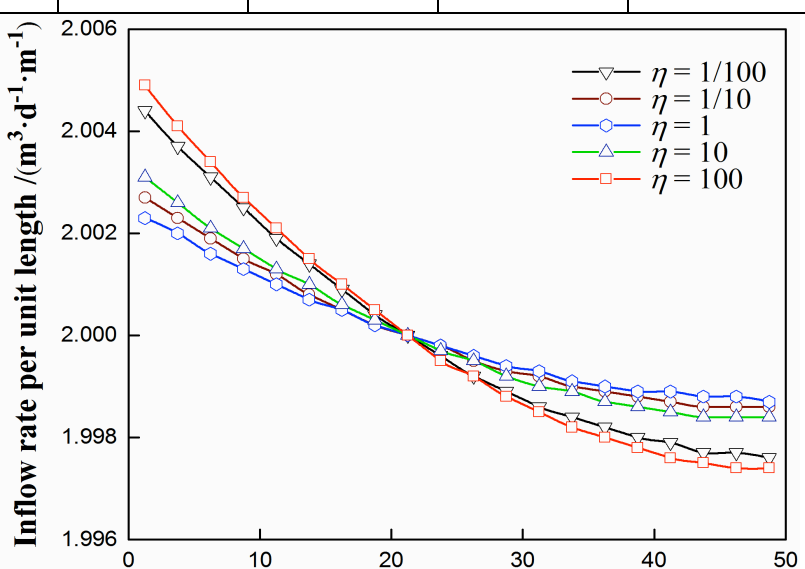

Distance from the heel of the wellbore $/ \mathrm{m}$

Fig. 4. Inflow distributions along the wellbore at $10^{-6} \mathrm{~d}$

When radial flow radius increases to a critical value, leakage flow areas at two end segments increase. As a result, inflow rates at two ends are higher than those in the central well segment, causing the boundary effect. Figure 5 shows an inclined U-shaped inflow distribution in the well located in the reservoir with a low anisotropy coefficient of $10^{-5} d$. The smaller the anisotropy coefficient, the earlier the inclined U-shaped inflow distribution happens.

Over time, inflow rates in well segments neighboring the wellbore first reduce as influenced by the top and bottom impermeable boundaries. Figure 6 shows the appearance of the $\mathrm{W}$-shaped flow section for the well located in the reservoir with anisotropy coefficient of 1/10. Furthermore, all segments will be influenced by top and bottom boundaries, and U-shaped inflow distribution will appear again, as shown in Fig. 7.

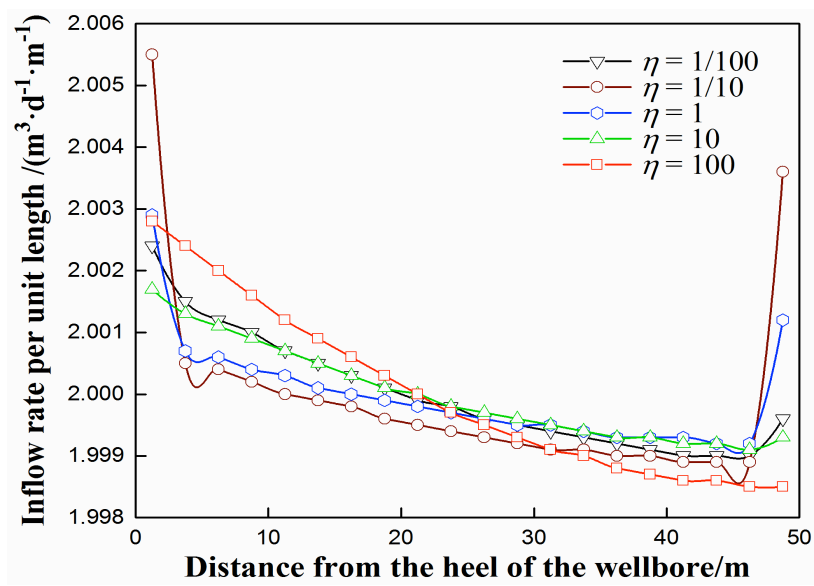

Fig. 5. Inflow distributions along the wellbore at $10^{-5} d$ 


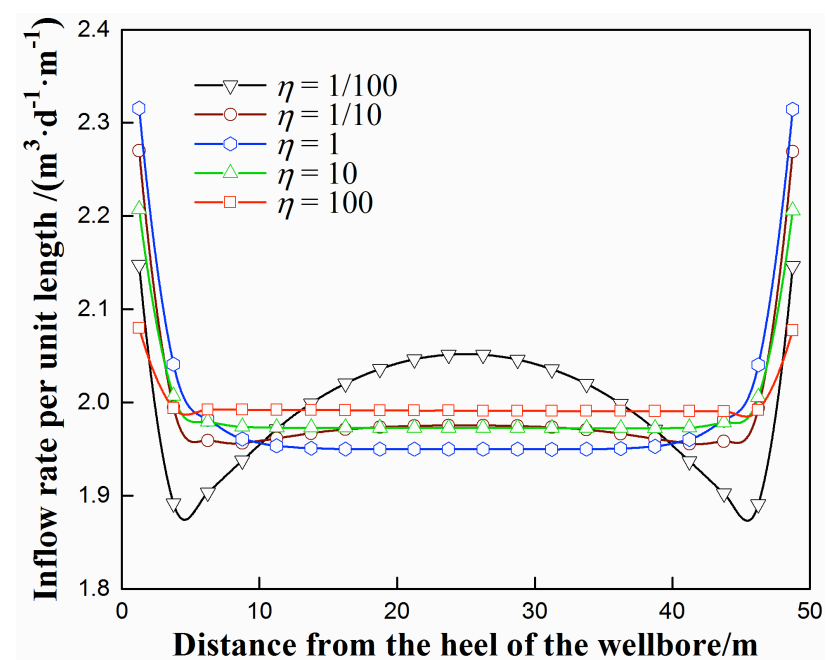

Fig. 6. Inflow distributions along the wellbore at $10^{-3} \mathrm{~d}$

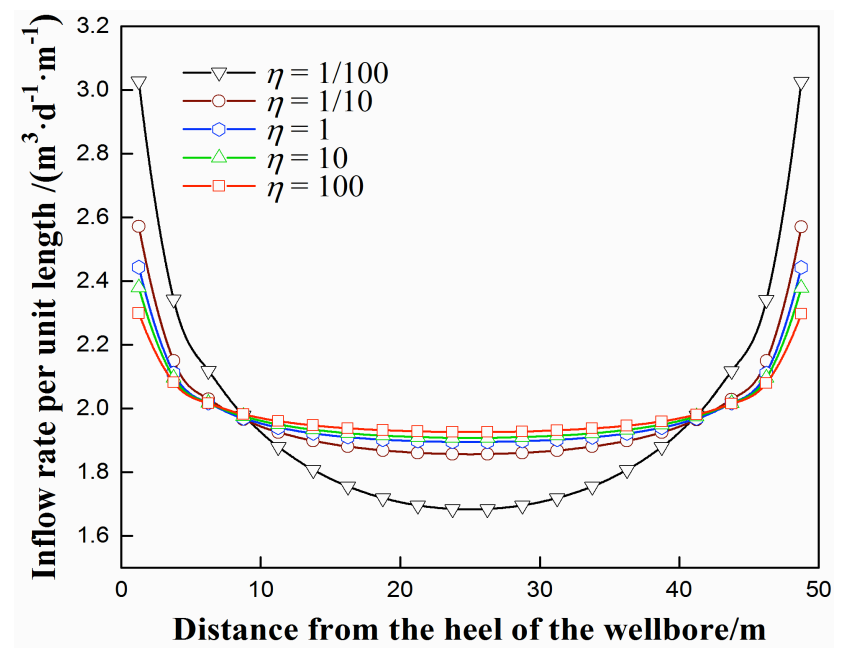

Fig. 7. Inflow distributions along the wellbore at $10^{-1} d$

The flow is approximate to a steady-state flow after approximately $10 \mathrm{~d}$, and inflow section tends to be steady. The potential distribution and inflow section are shown in Figs. 8 and 9 , respectively. Figure 8 shows that the well bottom potential increases as the anisotropy coefficient increases. Through a comparison with the potential section at $10^{-6} d$, potential drop is greatly aggravated, but the potential drop along the wellbore almost does not change. Thus, compared with early-stage flow, potential drop becomes relatively unimportant. Figure 9 indicates that the inflow difference between heel end and toe end caused by potential drop is very small. Figure 9 also shows that when the anisotropy coefficient is smaller than 1 , inflow rates in well segments nearby two ends significantly increase as the anisotropy coefficient decreases.

\subsection{Productivity}

The comprehensive influence of vertical anisotropy and well deviation angle on the yield under steady-state condition is shown in Fig. 10. Seven different well deviation angles of $0^{\circ}, 15^{\circ}, 30^{\circ}, 45^{\circ}, 60^{\circ}, 70^{\circ}, 75^{\circ}$, and $90^{\circ}$ were considered.

When the anisotropy coefficient is equal to 0.01 , the yield of the well with a large well deviation angle is slightly greater than that of the well with a smaller well deviation angle. The yield index increases as the anisotropy coefficient increases, but the larger the well deviation angle, the less the yield index increases. When a certain anisotropy coefficient threshold (slightly greater than 1) is reached, the yield index of the well with a small well deviation angle exceeds that of the well with a large well deviation angle. When the anisotropy coefficient is 100 , the yield index of the well with a small well deviation angle is clearly greater than that with a large well deviation angle.

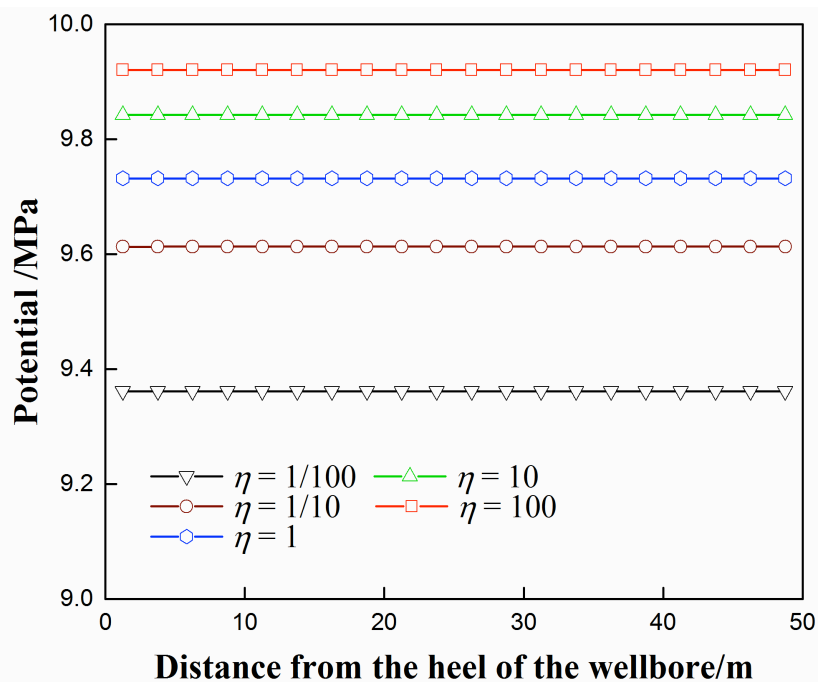

Fig. 8. Wellbore potential profiles under steady-state conditions

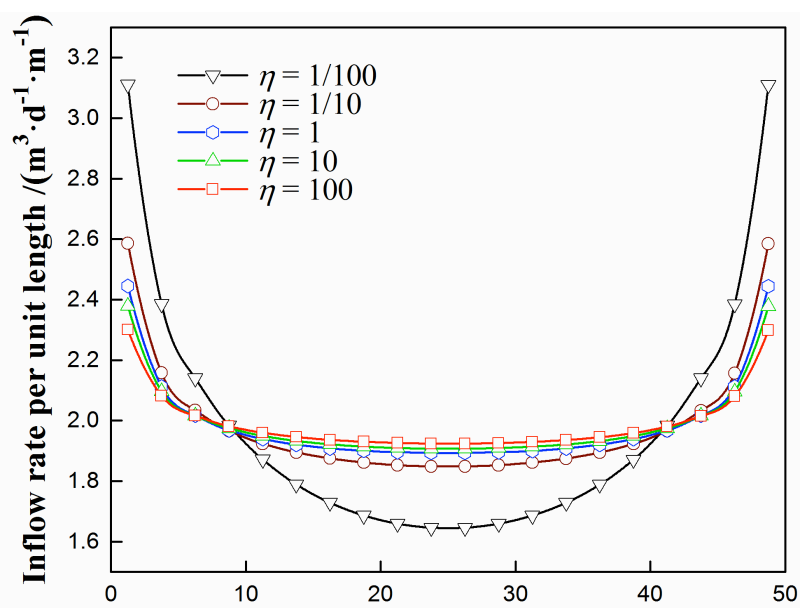

Distance from the heel of the wellbore/m

Fig. 9. Inflow distributions along the wellbore under steady-state conditions

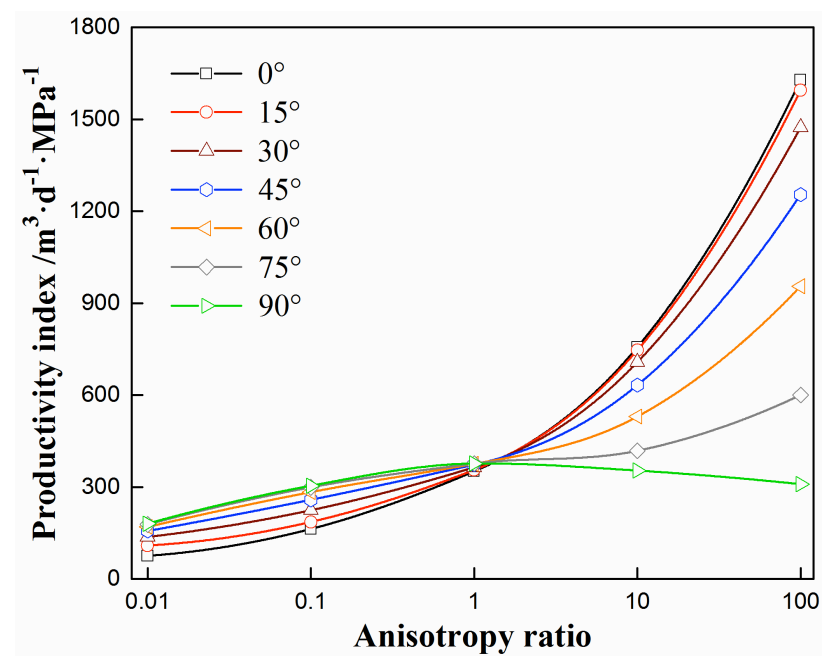

Fig. 10. Effects of vertical anisotropy and inclination angle on productivity 


\section{Conclusions}

To establish a high-efficiency inflow performance evaluation model for the highly deviated wells in the anisotropic reservoir, a semi-analytical method was employed in this study to analyze the inflow performance of highly deviated wells based on the reservoir-wellbore coupling flow model. The following conclusions were drawn:

(1) The established inflow performance evaluation model of the highly deviated well can guarantee calculation accuracy. The calculation time is only $1 / 30$ that of the traditional model, and it can significantly improve the analysis efficiency.

(2) Inflow morphology of the highly deviated well can be divided into three stages: early-stage radial flow, transitionstage transient flow, and later-stage steady flow. The inflow section of the highly deviated well mainly presents a " $U$ " shape, and flow velocities near the wellbore heel end and toe end are clearly higher than those in other positions.

(3) Reservoir anisotropy greatly influences the inflow performance of highly deviated wells. The smaller the anisotropy coefficient, the earlier the transient flow at the transition stage appears, and the later the steady flow at the later stage appears.

(4) The anisotropy coefficient apparently influences the flow velocity in the wellbore. When the coefficient increases from $1 / 100$ to 100 , inflow velocities at the two ends of the wellbore decrease by $32.3 \%$.

(5) The influence of well deviation angle on the yield of the highly deviated well is enhanced as the reservoir anisotropy coefficient increases. Thus, the influence of anisotropy features of the actual reservoir in must be comprehensively considered in the design of highly deviated wells.

A high-efficiency inflow performance evaluation model for the highly deviated well was proposed in this study. The established model can consider the influences of reservoir anisotropy and well deviation angle, and it is proven to be highly efficient. Moreover, similar with actual production, it can contribute to a better understanding of the inflow performance and performance of highly deviated wells. It can also be of a certain reference value to well completion scheme optimization design and productivity prediction of highly deviated wells. Due to the lack of field production data of highly deviated wells, field feedback data should be continuously combined to perfect and correct the model in future studies to enhance its applicability to reservoirs of different properties.

\section{Acknowledgements}

This work was supported by the National Science and Technology Major Project (Grants No: 2011ZX05006-002), and the National Natural Science Foundation of China (Grants No: 51804323).

This is an Open Access article distributed under the terms of the Creative Commons Attribution License

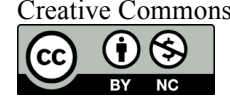

\section{References}

1. M. Jamiolahmady, P. Ghahri, O.E. Victor, et al. "Comparison of Vertical, Slanted, and Horizontal Wells Productivity in Layered Gas-Condensate Reservoirs". In: Europec/EAGE Annual Conference and Exhibition, Londong, United Kingdom: SPE, 2007, pp.212-217.

2. S. R. Shadizadeh, M. A. Zoveidavianpoor. "A Successful Experience in Optimization of a Production Well in a Southern Iranian Oil Field”. Iranian Journal of Chemical Engineering, 10(6), 2009, pp.37-49.

3. M. L. Szanyi, C.S. Hemmingsen, W. Yan, et al. "Near-Wellbore Modeling of a Horizontal Well with Computational Fluid Dynamics". Journal of Petroleum Science and Engineering, 160(2), 2018, pp.119-128.

4. G. Feng, Q. Liu. "Pressure Transient Behavior of a Slanted Well with an Impermeable Fault". Journal of Hydrodynamics, 26(B), 2015, pp.980-985.

5. H. Wang, L. Zhang, J. Guo, et al. "An Efficient Algorithm to Compute the Transient Pressure Responses of Slanted Wells with Arbitrary Inclination in Reservoirs". Petroleum Science, 40(9), 2012, pp.212-222.

6. P.R. Tsou, Z.Y. Feng, H.D. Yeh, et al. "Stream Depletion Rate with Horizontal or Slanted Wells in Confined Aquifers near a Stream". Hydrology and Earth System Sciences, 2010, 14(2): 1477-1485.

7. H. Zhan, V.A. Zlotnik. "Groundwater Flow to a Horizontal or Slanted Well in an Unconfined Aquifer". Water Resources Research, 38(1), 2002, pp.1108-1122.

8. X. Zhang, X. Li, X. Zhang. "Research on Inclined Well Test Model for Orthographic Fault Slab by Double Porosity Reservoir". Well Testing, 21(4), 2012, pp.1-4.

9. B. Vaferia, V. Salimib, D. Dehghan Banianic, et al. "Prediction of Transient Pressure Response in the Petroleum Reservoirs Using Orthogonal Collocation". Journal of Petroleum Science and Engineering, 98(4), 2012, pp.156-163.
10. D. Biryukov, F. J. Kuchuk. "Transient Pressure Behavior of Reservoirs with Discrete Conductive Faults and Fractures". Transport in Porous Media, 95(5), 2012, pp.239-268.

11. A. R. Bahadori, A. Jamili, S. Zendehboudi. "Calculating PseudoSteady-State Horizontal Oil Well Productivity in Rectangular Drainage Areas Using a Simple Method". Chemical Engineering Communications, 200(1), 2013, pp.222-234.

12. M. A. Ahmadi, R. Soleimani, M. Lee, et al. "Determination of Oil Well Production Performance Using Artificial Neural Network (ANN) Linked to the Particle Swarm Optimization (PSO) Tool". Petroleum, 1(2), 2015, pp.118-132.

13. M. Tabatabaei, D. Zhu. "Generalized Inflow Performance Relationships for Horizontal Gas Wells". Journal of Natural Gas Science and Engineering, 2(3), 2010, pp.132-142.

14. SHI Ting, GUO Xiao, TANG Line, et al. "A New Method to Predict Production of Horizontal Well in Bottom Water Reservoir". Petrochemical Industry Application, 35(2), 2016, pp.16-23.

15. Wang Yining, Li Hong, Cao Shuhui, et al. "Productivity Formula of Long Horizontal Well in Anisotropic Bottom-water Reservoir". Petroleum Geology and Recovery Efficiency, 23(4), 2016, pp.108116.

16. Chen Yuanqian, Guo Erpeng, Peng Zixuan, et al. "Derivation of Productivity Formulas for Horizontal Wells in an Isotropic faultblock Resrvoirs". ACTA PETROLEI SINICA, 38(12), 2017, pp.1420-1424.

17. Lei Gang, Dong Pingchuan, Rao Peiyu, et al. "Analysis of Pressure Characteristics of Oil Well in Anisotropic Fault-block Oil Reservoirs". Journal of Southwest Petroleum University (Science \& Technology Edition ) , 37(2), 2015, pp.125-130.

18. M. Soleimani, B. Jodeiri-Shokri, M. Rafiei. "Integrated Petrophysical Modeling for a Strongly Heterogeneous and Fractured Reservoir, Sarvak Formation, SW Iran”. Nature Resources Research, 26(1), 2017, pp.75-88. 
19. M. Delalat, R. Kharrat, "Investigating the Effects of Heterogeneity, Injection Rate and Water Influx on GAGD EOR in Naturally Fractured Reservoirs". Iranian Journal of Oil \& Gas Science and Technology, 2(1), 2013, pp.9-21.

20. D. E. Williams. "Drawdown Distribution in the Vicinity of Nonvertical Wells". Ground Water, 51(5), 2013, pp.745-751.

21. Wang Haijing, Xue Shifeng, Gao Cunfa, et al. "Inflow Performance for Highly Deviated Wells in Anisotropic Reservoirs". Petroleum Exploration and Development, 39(2), 2012, pp.222-227.
22. FANG Sidong, CHENG Linsong, XIN Yinan, et al. "Linear Element Method for Multi-angle Fractured Horizontal Well in Anisotropic Reservoir". Chinese Journal of Computational Physics, 32(5), 2015, pp.595-602.

23. REN Zongxiao, WU Xiaodong, HE Xiaojun, et al. "Model of Transient Pressure Behavior for Horizontal Wells with Inclined Fractures in Anisotropy Reservoir". Fault-block Oil \& Gas Field, 24(1), 2017, pp.74-78. 